\title{
Handgrip Strength Prediction Formula Using Aneroid Sphygmomanometer in Elderly
}

\author{
Akhmad Yahin, Marina Annette Moeliono, Marietta Shanti Prananta \\ Department of Physical Medicine and Rehabilitation, Faculty of Medicine, Universitas Padjajaran-Dr. Hasan \\ Sadikin General Hospital
}

$\begin{array}{ll}\text { Abstract } & \text { Objective: To examine the prediction formula used to measure handgrip } \\ \text { strength using aneroid sphygmomanometer in elderly. } \\ \text { Methods: This study was a cross-sectional study performed at the } \\ \text { Department of Physical Medicine and Rehabilitation, Faculty of Medicine, } \\ \text { Universitas Padjadjaran-Dr. Hasan Sadikin General Hospital from March to } \\ \text { April 2015. Thirty five elderly subjects aged 60-74 years were included in } \\ \text { the study. The subject handgrip strength was measured using an aneroid } \\ \text { sphygmomanometer and a Jamar dynamometer. } \\ \text { Results: All data were analyzed statistically using Pearson correlation test } \\ \text { to examine the distributed data and Spearman test to examine the non } \\ \text { distributed data. A significant correlation was found between hand width, } \\ \text { age, and sex and the handgrip strength values using Jamar dynamometer } \\ \text { (p<0.01) using multiple linear regression (p<0.01). A normality test using } \\ \text { the Shapiro-Wilk test resulted normal residue with p value 0.000. The results } \\ \text { showed that the regression analysis could be used to predict the handgrip } \\ \text { strength using aneroid sphygmomanometer. } \\ \text { Conclusions: Aneroid sphygmomanometer can be used to predict handgrip } \\ \text { strength in elderly using conversion formula. } \\ \text { Received: } \\ \text { November 2, 2015 } \\ \text { Revised: } \\ \text { July 17, 2016 }\end{array}$

\section{Introduction}

Ageing is as a gradual changing process in adults from healthy to weaker condition caused by decreased physiology system ability in the body. ${ }^{1}$ The physiological changes do not only influence the physical performances but also the physical functions and responses in performing daily life. Several distinctive changes in body systems caused by the ageing process are significant decreased of muscle mass and muscle strength.

Muscle strength in elderly is associated with several functional abilities. ${ }^{2}$ Functional abilities in elderly are defined as the abilities in performing daily activities integrated to their

Correspondence:

Akhmad Yahin, Department of Physical Medicine and Rehabilitation, Faculty of Medicine, Universitas Padjajaran-Dr. Hasan Sadikin General Hospital Jl. Pasteur No. 38, Bandung, Indonesia e-mail: akhmadyahin@outlook.com environments. The minor muscle weakness disturbs daily activities and decreases the quality of life. ${ }^{3}$ Muscle strength measurement can be performed as well as measuring the handgrip strength.

Hands are particularly used to grasp any object. There are three steps in grasping: open the hand, close the fingers to grasp the object, and control the grip power. ${ }^{4}$ The study also described two types of grip movements, power grip and prehension grip. The power grip needs maximal power such as grasping a hammer and picking a rope bag with fingers in the half maximum flexion position while the palm and the thumb are on the opposite side. The prehension grip requires main function of the hand such as grasping a cone-shaped object.

The weaker handgrip may decrease health condition. A study reported that weak handgrip in elderly became an indicator of 
health problems and increased mortality risks. ${ }^{5}$

The handgrip strength can be measured by using a specific medical apparatus, a handgrip dynamometer. One of most used handgrip dynamometers with a typical hydraulic system is namely Jamar dynamometer as described in this study (Fig. 1a, b, c). Alternatively, handgrip strength can be measured using another apparatus, it is aneroid sphygmomanometer (Fig. 1d). Aneroid sphygmomanometer is an apparatus to measure blood pressure becoming the gold standard in blood pressure measurement. ${ }^{6-8}$

This study aimed to measure the handgrip strength in elderly. The study was initiated to
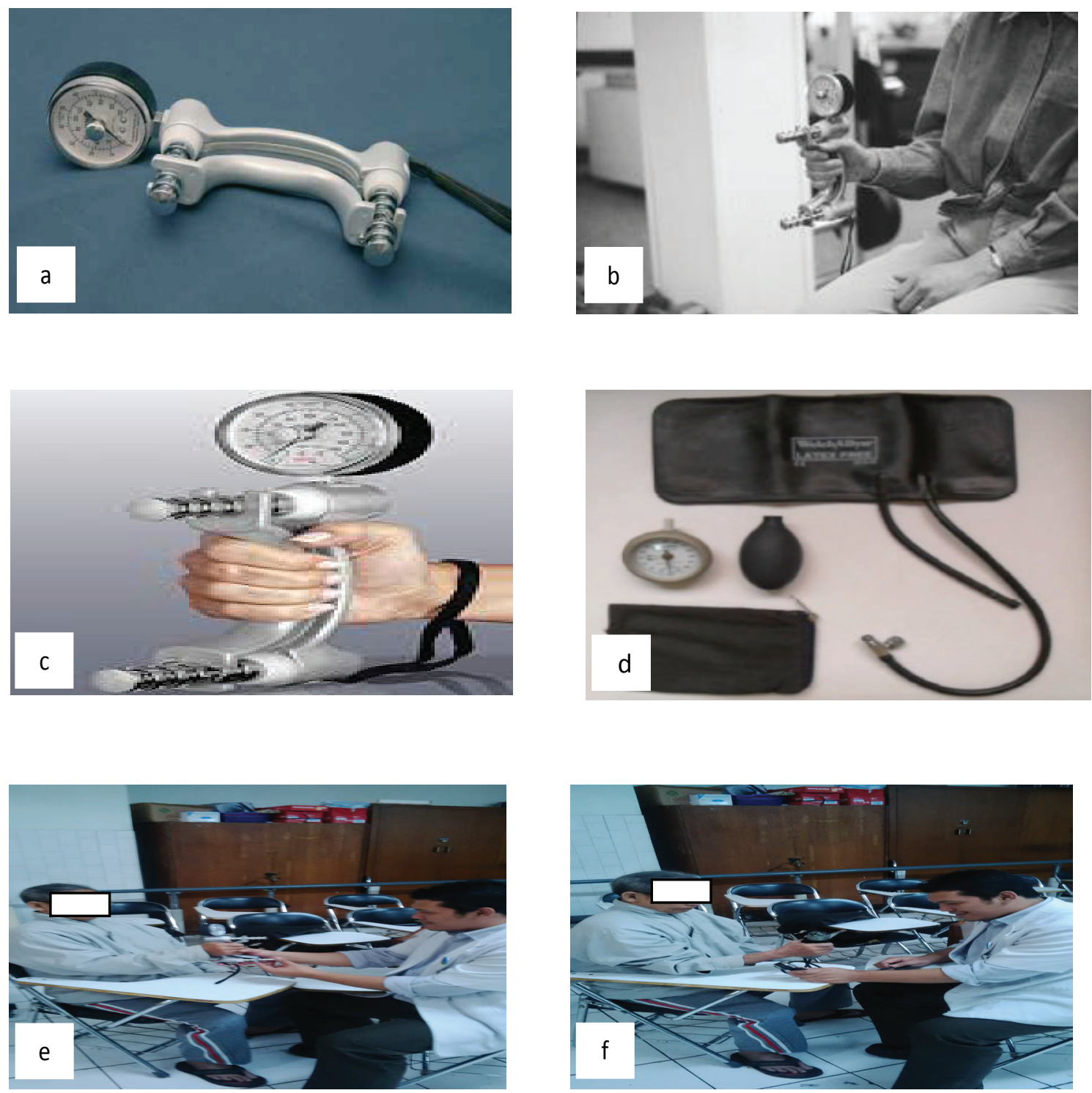

Fig. 1 Handgrip Jamar Dynamometer, 1a. ${ }^{10}$ 1b, Body Position in Grasping Jamar Dynamometer. $^{16}$ 1c, Hand Position in Grasping Jamar Dynamometer. ${ }^{16}$ 1d, Aneroid Sphygmomanometer. ${ }^{17}$ 1e, The Subject Handgrip Strength was Measured Using Jamar Dinamometer. 1f, The Subject Handgrip Strength was Measured Using aneroid Sphygmomanometer 
Akhmad Yahin, Marina Annette Moeliono, et al.

Table 1 Subject Characteristics

\begin{tabular}{lccccc}
\hline \multirow{2}{*}{ Variable } & \multicolumn{2}{c}{ Male } & \multicolumn{2}{c}{ Female } & \multirow{2}{*}{ p Value } \\
\cline { 2 - 5 } & Average & Median & Average & Median & \\
\hline Age & & $65(61-74)$ & & $63.5(61-74)$ & 0.3840 \\
Height & $166(161-171)$ & & $155(150-168)$ & 0.0003 \\
Weight & $63.07(6.713)$ & & $56.54(7.570)$ & & 0.0149 \\
Middle finger length & & $16(15-19.5)$ & & $16(14-19)$ & 0.2527 \\
Hand width & & $8.5(7-1)$ & & $7.75(6-10)$ & 0.0342 \\
Handgrip diameter & & $14.5(13-18.5)$ & & $14(13-18)$ & 0.1357 \\
Right sphyg* & $172.46(10.55)$ & & $133.90(27.12)$ & & 0.0000 \\
Left sphyg* & $161.15(11.57)$ & & $124.50(27.90)$ & & 0.0000 \\
Right Jamar & & $37(21-50)$ & & $16.25(12-26)$ & 0.0000 \\
Left Jamar & & $35(20-47.5)$ & & $14(11-23)$ & 0.0000 \\
\hline
\end{tabular}

Notes

Right sphyg = Right handgrip strength measurement using aneroid sphygmomanometer

Left sphyg = Left handgrip strength measurement using aneroid sphygmomanometer

Right Jamar = Right handgrip strength measurement using Jamar dynamometer

Left Jamar = Left handgrip strength measurement using Jamar dynamometer

estimate the prediction formula to measure the handgrip strength by using aneroid sphygmomanometer and Jamar dynamometer. The prediction formula becomes the most effective effort to measure elderly handgrip strength.

\section{Methods}

This study was conducted at the Department of Physical Medicine and Rehabilitation, Faculty of Medicine, Universitas PadjadjaranDr. Hasan Sadikin General Hospital in MarchApril 2015. This study was approved by the Health Research Ethic Committee, Faculty of Medicine, Universitas Padjadjaran with ethic number: LB.04.01/A05/EC/093/IV/2015.

The subjects of this study were patients who attended Physical Medicine and Rehabilitation Polyclinic, Dr. Hasan Sadikin General Hospital, Bandung who met the inclusion and exclusion criteria. The inclusion criteria were aged 6074 years, able to comprehend the instructions with mini mental status examination (MMSE) score $\geq 24$, cooperative and willing to be included in the study. The exclusion criteria were patients with sensibility and deformity problems in their upper body.

The subjects involved in the study were
35 healthy elderly (13 males and 22 females) and calculated by consecutive sampling. All subjects lived in Bandung, Indonesia, and willing to be included in the study after signing the informed consent.

The subjects were recruited based on their medical records and physical characteristics, including subject body weight and height. Hand length was measured from the distal ulnar wrist crease to the end of middle finger while the hand and all the fingers opened. Hand width was measured from the distal palmar crease to the radial distal palmar crease while the hand was on straight position. Then, the subjects were instructed to grasp a coneshaped object while the thumb met the middle finger to measure the handgrip diameter.

Thesubjecthandgripstrengthwas measured using Jamar Hydrolic hand dynamometer type J00105 (Lafayette Instrument Company, Sagamore Parkway North, Lafayette, USA) and One Med Aneroid Sphymomanometer type T-200 (PT. Jayamas Medica Industri, Sidoarjo, Indonesia) with participants seated, 90 degrees elbow flexion (Fig 1e). Value of handgrip strength was displayed on the screen in kilogram meter. The right and left handgrip strengths were measured three times. Between each measurement took 5 minutes rest to prevent fatigue. Results were obtained 
from the mean value after three trials.

The subject handgrip strength was then measured using aneroid sphygmomanometer (Fig. 1d). Several instructions were asked to all subjects to grasp the rolled cuff with the wrist in neutral position. The aneroid sphygmomanometer level should be adjusted to $20 \mathrm{mmHg}$. The right and left handgrip strengths were measured three times with 5 minutes rests for each measurement. The

Table 2 Multivariable Correlation Test to Handgrip Strength Value

\begin{tabular}{lcc}
\hline \multicolumn{1}{c}{ Variable } & r (n=35) & p Value \\
\hline Right sphyg and weight* & 0.3550 & 0.036 \\
Left sphyg and weight* & 0.3216 & 0.060 \\
Right sphyg and age & -0.012 & 0.947 \\
Right sphyg and sex & 0.6863 & 0.0000 \\
Right sphyg and heigth & 0.407 & 0.0153 \\
Right sphyg and middle finger length & 0.547 & 0.0007 \\
Right sphyg and hand width & 0.578 & 0.0003 \\
Right sphyg and handgrip diameter & 0.569 & 0.0004 \\
Right sphyg and Right Jamar & 0.825 & 0.0000 \\
Right sphyg and left Jamar & 0.886 & 0.0000 \\
Left sphyg and age & -0.016 & 0.9267 \\
Left sphyg and sex & 0.6725 & 0.0000 \\
Left sphyg and heigth & 0.411 & 0.0142 \\
Left sphyg and middle finger length & 0.509 & 0.0018 \\
Left sphyg and hand width & 0.578 & 0.0002 \\
Left sphyg and handgrip diameter & 0.521 & 0.0013 \\
Left sphyg and Right Jamar & 0.833 & 0.0000 \\
Left sphyg and Left Jamar & 0.897 & 0.0000 \\
Right Jamar and age & -0.1475 & 0.9640 \\
Right Jamar and heigth & 0.3349 & 0.0492 \\
Right Jamar and weigth & 0.2023 & 0.0437 \\
Right Jamar and age & 0.7520 & 0.0000 \\
Right Jamar and hand width & 0.6164 & 0.0000 \\
Left Jamar and age & -0.116 & 0.9345 \\
Left Jamar and heigth & 0.4599 & 0.0054 \\
Left Jamar and weigth & 0.3184 & 0.0623 \\
Left Jamar and sex & 0.7962 & 0.0000 \\
Left Jamar and hand width & 0.5938 & 0.0002 \\
\hline & & \\
& & \\
& &
\end{tabular}

Notes

Right sphyg = Right handgrip strength measurement using aneroid sphygmomanometer

Left sphyg = Left handgrip strength measurement using aneroid sphygmomanometer

Right Jamar = Right handgrip strength measurement using Jamar dynamometer

Left Jamar = Left handgrip strength measurement using Jamar dynamometer

*Pearson correlation test, other data used Spearrman test 
Akhmad Yahin, Marina Annette Moeliono, et al.

Table 3 Coefficient Regression Analysis and p Value to Measure Right Handgrip Strength Using Jamar Dynamometer

\begin{tabular}{lcccccc}
\hline & BETA & $\begin{array}{c}\text { St.Err. of } \\
\text { BETA }\end{array}$ & B & St.Err. of B & t(31) & p Value \\
\hline Right hand & & & & & & \\
$\quad$ Right sphyg & 0.6255 & 0.2373 & 0.1123 & 0.0426 & 2.6359 & 0.013 \\
Sex & 0.2308 & 0.0538 & 10.2679 & 2.3947 & 4.2877 & 0.0002 \\
Hand width & 0.7938 & 0.2766 & 2.6346 & 0.918 & 2.8699 & 0.0073 \\
Age & -0.6214 & 0.2521 & -0.2563 & 0.104 & -2.4654 & 0.0194 \\
Left hand & & & & & & \\
$\quad$ Left sphyg & 0.7386 & 0.0519 & 0.1306 & 0.0092 & 14.2426 & 0.0000 \\
Sex & 0.3063 & 0.0519 & 12.5348 & 2.1221 & 5.9067 & 0.0000 \\
\hline
\end{tabular}

Notes

Right sphyg = Right handgrip strength measurement using aneroid sphygmomanometer

Left sphyg = Left handgrip strength measurement using aneroid sphygmomanometer

value after three times trials were taken.

This was an observational study using correlational test and also cross-sectional analysis to examine the prediction formula to measure the handgrip strength using aneroid sphygmomanometer. The results were conversion formula from the measurement values using aneroid sphygmomanometer compared to the measurement values using Jamar dynamometer due to estimate the handgrip strength. This study analyzed two variables, dependent and independent variables.

The dependent variable was measurement values using Jamar dynamometer, while the independent variable was the measurement values using aneroid sphygmomanometer and hand anthropometry results (hand length, width, and diameter).

The two variables were examined using Spearman and Pearson correlation tests, however, the examination depended on the data distribution. The simple linear regression and multiregression analyses were peformed by adding hand anthropometry results and sex as additional independent variables (dummy variables).

Shapiro Wilk test was performed due to the total subjects were $<50$. The results showed that the subject characteristics such as weight and right and left handgrip strength values using aneroid sphygmomanometer were normally distributed. The t test was performed to analyze the comparison between male and female subjects' characteristics. Non normally distribution were found in age, height, middle finger length, hand width, handgrip diameter, and right and left handgrip strength values using aneroid sphygmomanometer. Mann Whitney test was performed to analyze the comparison between male and female subjects' characteristics.

The data were the handgrip strength measurementvaluesusingJamardynamometer and aneroid sphygmomanometer. Then, values were combined with the anthropometry results. The combination generated the conversion formula of handgrip strength measurement using aneroid sphygmomanometer.

\section{Results}

Thirty five healthy participants, 13 males and 22 females, were assesed for eligibility and performed all measurement with the same method to collected all data (Fig. 2). This study analyzed the subject characteristics compared to handgrip strength measurement values. Statistical examination using t-test showed significant difference between subject characteristics, including weights $(\mathrm{p}=0.0149)$, handgrip strength values using aneroid sphygmomanometer on right $(\mathrm{p}=0.0000)$ and left $(\mathrm{p}=0.0000)$ hands (Table 1$)$.

Significant differences were found using Mann Whitney test in analyzing subject weight $(\mathrm{p}=0.0003)$, hand width $(\mathrm{p}=0.0342)$, 
and handgrip strength values using Jamar dynamometer on right $(\mathrm{p}=0.0000)$ and left $(\mathrm{p}=0.0000)$ hands. No significant difference was found using Mann Whitney test in analyzing male and female subjects in age $(p=0.3840)$, middle finger length $(p=0.2527)$, and handgrip diameter $(\mathrm{p}=0.1375)$.

The correlation analysis was performed between the measurement values using Jamar dynamometer and the measurement values using aneroid sphygmomanometer compared to the variables (Table 2). The multivariable correlation test was performed using Spearman correlation test to analyze the normal distributed data, while the Pearson correlation test was used to analyze the nondistributed data. A significant correlation was found in the related variables, except the age variable when compared to the handgip strength measurement values using aneroid sphygmomanometer and Jamar dynamometer (Table 2). The correlational analysis among the variables resulted different correlation values.

The correlational test between ages and the handgip strength measurement values using aneroid sphygmomanometer and Jamar dynamometer were not considered statistically significant. The correlational test between weights and handgrip strength measurement values using aneroid sphygmomanometer and Jamar dynamometer of right and left hands was not considered statistically significant $(\mathrm{r}=0.2-0.4)$.

The correlation test between the subject height, middle finger length, and hand width compared to thehandgipstrengthmeasurement values using aneroid sphygmomanometer and Jamar dynamometer of right and left hands were considered statistically moderate $(r=0.4-0.6)$. The correlation test between sex and the handgip strength measurement values using aneroid sphygmomanometer and Jamar dynamometer of right and left hands was considered statistically significant $(\mathrm{r}=0.6-0.8)$.

A regression test were performed between handgip strength measurement values using Jamar dynamometer and several variables, including right handgip strength aneroid sphygmomanometer measurement values, sex, hand width, dan age (Table 3). The results revealed that the related variables might influence significantly compared to the right handgrip strength Jamar dynamometer measurement values. The results showed that the left handgip strength measurement values using aneroid sphygmomanometer and sex influenced significantly different compared to left handgip strength Jamar dynamometer measurement values ( $p<0.05$ ) (Table 3 ).

To generate the regression analysis, residual test was performed to find the residual mean and residual distribution.

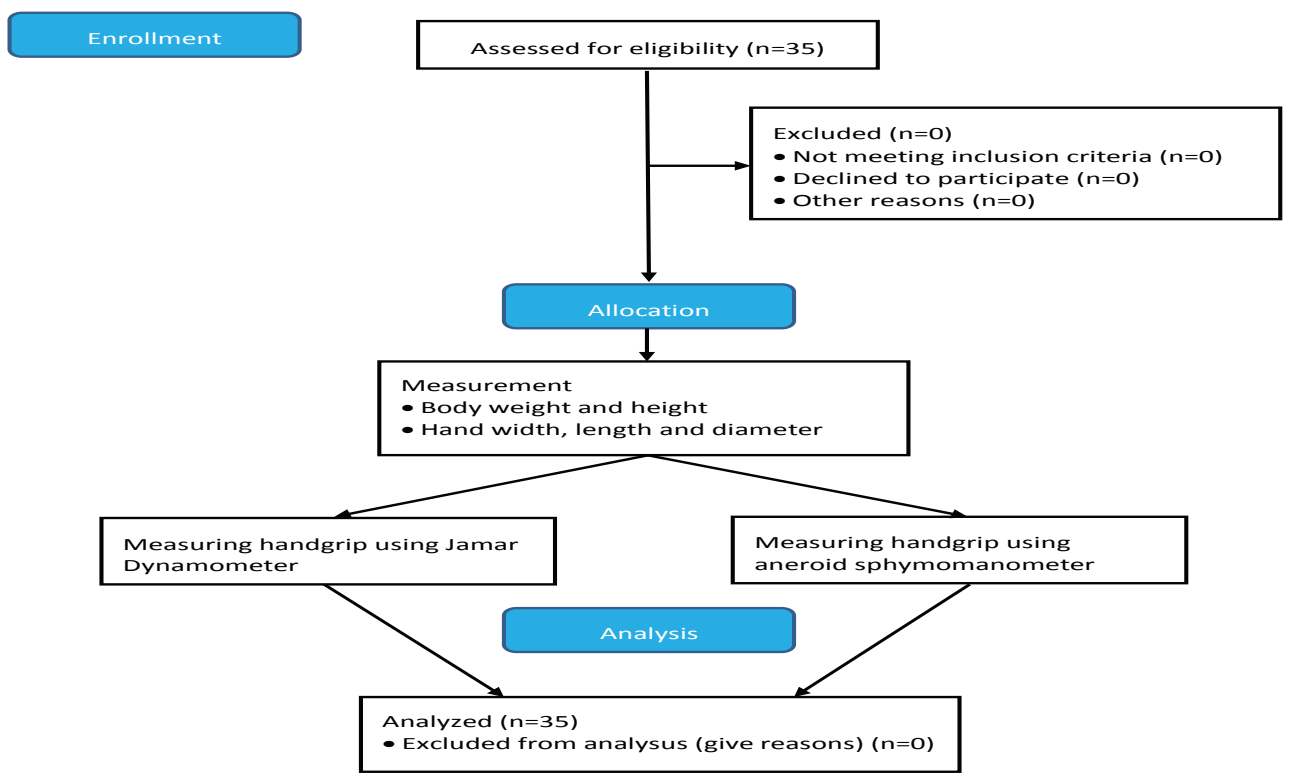

Fig. 2 Flowchart of Patient Recruitment 
Table 4 Coefficient Regression Analysis and p Value to Measure Right and Left Handgrip Strength Using Jamar Dynamometer

\begin{tabular}{lcccccc}
\hline & BETA & $\begin{array}{c}\text { St.Err. of } \\
\text { BETA }\end{array}$ & B & St.Err. of B & $\mathbf{t}(\mathbf{3 3})$ & p Value \\
\hline Right Jamar & & & & & & \\
Right sphyg & 0.6255 & 0.2373 & 0.1123 & 0.0426 & 26.359 & 0.0130 \\
Sex & 0.2308 & 0.0538 & 102.679 & 23.947 & 42.877 & 0.0002 \\
Hand Width & 0.7938 & 0.2766 & 26.346 & 0.9180 & 28.699 & 0.0073 \\
Age & -0.6214 & 0.2521 & -0.2563 & 0.1040 & -24.654 & 0.0194 \\
Left Jamar & & & & & & \\
Left sphyg & 0.5342 & 0.2220 & 0.0883 & 0.0367 & 24.059 & 0.0233 \\
Sex & 0.3006 & 0.0504 & 126.581 & 21.214 & 59.668 & $2.31 \mathrm{E}-06$ \\
Hand Width & 0.9201 & 0.3293 & 26.562 & 0.9507 & 27.938 & 0.0095 \\
Age & -0.6880 & 0.2725 & -0.2381 & 0.0943 & -25.249 & 0.0178 \\
\hline
\end{tabular}

Notes

Right Sphyg = Right handgrip strength measurement using aneroid sphygmomanometer

Left Sphyg = Left handgrip strength measurement using aneroid sphygmomanometer

Right Jamar $=$ Right handgrip strength measurement using Jamar dynamometer

Left Jamar = Left handgrip strength measurement using Jamar dynamometer

Therefore, the residual mean was considered statistically significant. The t-test was performed to examine the residual value $=0$, and the value was 15.79 ( $\mathrm{p}$ value $>0.05$ ). The value was not considered valid to generate the handgrip strength prediction formula. The invalid value determined that further analyses should be performed. The error was caused by the presence of outlier data. There were four data which were considered invalid because of higher values.

The outlier data were not included in the subsequence regression analysis. The results showed a new coefficiet regression table to measure the handgrip strength values of left hand using Jamar dynamometer (Table 4).

The handgip strength using aneroid sphygmomanometer values of right and left hands, sex, hand width, dan age influenced significantly compared to the handgrip strength Jamar dynamometer measurement values of right hand.

After eliminating the outlier data, the coefficient regression results showed handgrip strength using aneroid sphygmomanometer values of left hand, sex, hand width, dan age influenced significantly compared to the handgrip strength Jamar dynamometer values of left hand ( $p$ value <0.05) (Tabel 4).
After generating the new coefficient regression analysis in several variables, new residual test was performed to discover residual mean and residual distribution. Then, the residual test results were found after eliminating the outlier data.

Normality test using Saphiro Wilk showed that the residue distributed normally and resulted the mean $=0$ and $p$ value was 0.0000 . It reveals that the regression analysis results could be generated to predict the handgrip strength values using Jamar dynamometer.

Based on regression analysis, the prediction formula resulted the handgrip strength values using aneroid sphygmomanometer (Table 4). The handgrip strength could be measured by using prediction formula.

Right Handgrip Strength Prediction Formula:

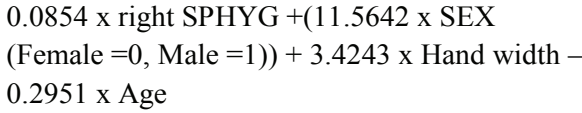

Left Handgrip Strength Prediction Formula: $0.0883 \times$ Left $S P H Y G+(12.6581 \times$ Sex $($ Female $=0$, Male $=1))+2.6562 \times$ Hand width $-0.2281 \times$ Age

Data were statistically examined and resulted the handgrip strength measurement 
prediction formula. The prediction formula were appropriately used to measure the handgrip strength in elderly.

\section{Discussion}

Handgrip strength is one of main factors related to hand functions. Several studies stated that there were many factors influence the handgrip strength such as age, race, hand domination, height, weight, and hand anthropometry. $5,8,9$

Participants in this study could understand the instruction during the test (MMSE score more than 24). All subjects in this study had similar hand characteristics which meant male and female subjects were not significantly different. The hand middle finger lengths in male and female subjects had the same median $(16 \mathrm{~cm})$ and handgrip diameters $(14 \mathrm{~cm})$ with the same average values. In this study, hand antrhopometry values were lower than the previous study.10 In literatures stated that hand middle finger length were $18 \mathrm{~cm}$ in males and $16 \mathrm{~cm}$ in females while the handgrip diameters were $15 \mathrm{~cm}$ in male subjects and 14 $\mathrm{cm}$ in female.

It showed that handgip strength values using aneroid sphygmomanometer and Jamar dynamometer of right and left hands were considered significantly different (Table 1). The handgrip strength of right hand were stronger than left hand. This study finds the total subjects had stronger and more dominant right hands.

In correlation analysis between related variables and the handgip strength values using aneroid sphygmomanometer and Jamar dynamometer showed positive correlation, except age variable. The age variable caused negative correlation that showed the opposite value compared to the handgip strength values using aneroid sphygmomanometer and Jamar dynamometer. The results were similar to the previous studies which reported that the handgrip strength was influenced by the subject age. ${ }^{2,5}$

The correlation analysis between the handgip strength values using aneroid sphygmomanometer and Jamar dynamometer and age was not considered statistically significant because the subject average age was $60-70$ years old. The results reveals that there was no significant correlation between the handgrip strength values and age. This study was different from the previous study which stated that no significant correlation between age and handgip strength measurement values. ${ }^{11}$

In correlation analysis between weight and height compared to the handgip strength values using aneroid sphygmomanometer and Jamar dynamometer were not considered statistically significant. The results were similar to the previous studies which revealed that weight was not the only factor influenced the handgrip strength values. ${ }^{10-12}$ Other factors that will influence the handgrip strength values were height and body mass index (BMI). However, this study was in agreement with the previous study which reported significant results between the handgip strength values using aneroid sphygmomanometer and Jamar dynamometer. ${ }^{7}$ The significant results were caused by the same handgip strength measurement movements between aneroid sphygmomanometer and Jamar dynamometer.

Regression test revealed that handgrip strength was influenced by sex. In the handgrip strength prediction formula, the different coefficient results between male and female subjects revealed that males are stronger than females.

A study reported that males had stronger handgrip than females. ${ }^{13}$ This different handgrip strength between males and females was caused by the muscle mass. Regression test in several variables and $p$ value revealed that hand width significantly influenced handgrip strength values (Table 4). The results were similar to the previous study which stated that significant correlation was found between hand anthropometry and handgrip strength measurement values. $7,8,15$ The studies described that an individual with wider hand had higher handgrip strength values.

The limitation of this study is in handgrip strength prediction formula. The prediction formula in this study can only be used to measure handgrip strength in 60-74 years elderly. The handgrip strength prediction formula cannot be used in both adolescent and adult. subjects due to the value differences and ineffective results. A further study may include the subject physical activities to obtain more accurate results. In conclusion, the inexpensive way to measure the handgrip strength in elderly is by using aneroid sphygmomanometer. 


\section{References}

1. Dietzel R, Felsenberg D, Armbrecht G. Mechanography performance tests and their association with sarcopenia, falls and impairment in the activities of daily living-a pilot cross-sectional study in 293 older adults. J Musculoskelet Neuronal Interact. 2015;15(3):249-56.

2. Teimoori A, Kordi MR, Choobine S, Heidari B. The effects of aging on muscle strength and functional ability of healthy iranian males. WJ Sport Sci. 2009;2(2):261-5.

3. Lum PS, Mulroy S, Amdur RL, Requejo P, Prilutsky BI, Dromerick AW. Gains in upper extremity function after stroke via recovery or compensation. Top Stroke Rehabil. 2009;16(4):237-53.

4. Perez MA, Rothwell JC. Distinct influence of hand posture on cortical activity during human grasping. J Neurosci. 2015;25(12):4882-9.

5. Ling CH, Taekema D, de Craen AJ, Gussekloo J, Westendorp RG, Maier AB. Handgrip strength and mortality in the oldest old population: the Leiden 85-plus study. CMAJ. 2010;182(5):42936.

6. Ma Y, Temprosa M, Fowler S, Prineas RJ, Montez MG, Brown-Friday J, et al. Evaluating the accuracy of an aneroid sphygmomanometer in a clinical trial setting. Am J Hypertens. 2009;22(3):263-6.

7. Hamilton GF, McDonald C, Chenier TC. Measurement of grip strength: validity and reliability of the sphygmomanometer and jamar grip dynamometer. J Orthop Sports Phys Ther. 1992;16(5):215-9.

8. Fallahi AA, Jadidian AA. The effect of hand dimensions, hand shape and some anthropometric characteristics on handgrip strength in male grip athletes and non-athletes. J Hum Kinet. 2011;29:151-9.
9. Kamarul T, Ahmad TS, Loh WY. Hand grip strength in the adult Malaysian population. J Orthop Surg (Hong Kong). 2006;14(2):172-7.

10. Chuan TK, Hartono M, Kumar N. Anthropometry of the Singaporean and Indonesian populations. Int J Indust Ergonom. 2010;40(6):757-66.

11. Spruit MA, Sillen MJ, Groenen MT, Wouters EF, Franssen FM. New normative values for handgrip strength: results from the UK Biobank. J Am Med Dir Assoc. 2013;14(10):5-11.

12. Koley S, Kaur SP. Correlations of handgrip strength with selected hand-armanthropometric variables in Indian interuniversity female volleyball players. Asian J Sports Med. 2011;2(4):220-6.

13. Putrawan IBP, Kuswardhani RAT. Faktor-faktor yang menentukan kekuatan genggam tangan pada pasien lanjut usia di panti wredha tangtu dan Poliklinik Geriatri RSUP Sanglah Bali. J Peny Dalam. 2011;12(2):87-91.

14. Park SW, Goodpaster BH, Strotmeyer ES, de Rekeneire N, Harris TB, Schwartz AV, et al. Decreased muscle strength and quality in older adults with type 2 diabetes: the health, aging, and body composition study. Diabetes. 2006;55(6):1813-8.

15. Mandahawi N, Imrhan S, Al-Shobakia S, Sarder B. Hand anthropometry survey for the Jordanian population. Int J Indust Ergonom. 2008;38(11):966-76.

16. Susan L. Michlovitz. Principles of hand theraphy. In: hand surgery. $1^{\text {st }}$ ed. Philadelphia: Lippincott Williams \& Wilkins; 2004.

17. Souza LA, Martins JC, Moura JB, TeixeiraSalmela LF, De Paula FV, Faria CD. Assessment of muscular strength with the modified sphygmomanometer test: what is the best method and source of outcome values? Braz J Phys Ther. 2014;18(2):191-200. 